\title{
REFLEXIONES SOBRE EL PERFIL PROFESIONAL PARA LA DIRECCIÓN DE BIBLIOTECAS UNIVERSITARIAS
}

\section{REFLECTIONS ON THE PROFESSIONAL PROFILE IN POSITIONS OF ACADEMIC LIBRARIES DIRECTOR}

\author{
José Pablo Gallo León \\ Universidad de Alicante. España/Spain \\ ipablo.gallo@ua.es
}

Recibido/Received: 17/02/2016

Modificado/Modified: 8/07/2016

Aceptado/Accepted: 6/09/2016

\section{RESUMEN}

La transformación del paradigma bibliotecario en la última década conlleva que los profesionales de la materia deban adquirir una serie de competencias nuevas, así como disponer de unas habilidades específicas cada vez más exigentes. Esto se hace especialmente crítico en el caso de los puestos de gestión, responsables de la toma de decisiones bajo la presión de un entorno cambiante y hostil. Por ello, se debería asegurar que las personas que accedan a dichos puestos respondan a un perfil profesional que les capacite para su desempeño. Se realiza, pues, una propuesta de competencias y habilidades con las que todo director de biblioteca universitaria debería contar.

\section{PALABRAS CLAVE}

Perfil del bibliotecario, director de biblioteca, bibliotecas universitarias, gestor público, directivo público, selección, dirección de biblioteca.

\section{SUMARIO}

1. Introducción. 2. Metodología y objetivos. 3. El director de biblioteca como gestor público. 4. El director de biblioteca como bibliotecario y como gestor de biblioteca. 5. Propuesta de perfil de director de biblioteca universitaria basada en competencias. 6. Conclusiones y propuestas de desarrollo de la investigación. Bibliografía. Anexo

\begin{abstract}
The transformation of the library paradigm in the last decade means that their professionals must acquire new skills and improve their increasingly demanding specific skills. This is especially critical in the case of management positions that are responsible for making decisions under the pressure of a changing and hostile environment. Therefore, it should be ensured that people accessing these positions correspond to a professional profile that enables them to perform properly. A proposal of competencies and skills that every director of university library should have is thus presented.
\end{abstract}

\section{KEYWORDS}

Librarian profile, library director, dean of libraries, academic libraries, public administrator, public management, recruitment. 


\section{CONTENTS}

1. Introduction. 2. Methodology and objectives. 3. Library director as a public manager. 4. Library director as a librarian and as a library manager. 5. Proposal for a competency-based professional profile in positions of academic library director. 6. Conclusions and proposals for research development. References. Annex.

\section{INTRODUCCIÓN}

Los bibliotecarios en puestos directivos están sometidos hoy a una exigencia mayor que nunca, en un entorno de doble crisis. Primero, la económica, que ha supuesto una drástica reducción presupuestaria, pérdida de personal e incluso cierre de algunos centros y sucursales. Después, la social y bibliotecaria, con un cambio de época hacia una sociedadred, basada en la interconectividad y en el flujo e intercambio de información. Esto lleva a un cambio de paradigma bibliotecario que exige de sus profesionales la renovación de sus competencias y habilidades. Al tiempo, se necesitan bibliotecarios con una capacidad de gestión y de negociación, además de con una gran visión estratégica; que también es necesaria ante los desafíos de las transformaciones tecnológicas, educativas y, por tanto, de los servicios bibliotecarios (Gallo-León, et al., 2014). Se debería asegurar que la dirección de las bibliotecas responda a personas con este perfil.

La inquietud por el liderazgo en estos tiempos de crisis se ve reflejada por una relativamente amplia bibliografía internacional, hasta el punto de que en los Estados Unidos (EUA) incluso se plantean programas de doctorado orientados especialmente al liderazgo gerencial en bibliotecas e instituciones de información, como en el conocido Simmons College (Hernon \& Schwartz, 2008). Además, la preocupación por la definición de las competencias necesarias para ejercer la profesión viene de lejos. "Los bibliotecarios han listado, debatido, revisado y negociado listas de competencias por 125 años, desde el inicio de la educación bibliotecaria formal" (Helmick \& Swigger, 2006: 62).

Este interés ha tenido una menor repercusión en el panorama nacional, donde sí ha habido mucho interés por el perfil del bibliotecario en general. Esto quizás se deba a que el acceso a los puestos de dirección de biblioteca suelen estar sujetos al procedimiento de libre designación, lo cual permite obviar el perfil profesional sustituyéndolo por la cercanía personal, la antigüedad o los balances de poder dentro de la política universitaria. Ciertamente son puestos de confianza, pero también debe ser necesario asegurar la adecuación al mismo.

Por otra parte, en los casos en el que el procedimiento de provisión es el de concurso se detecta la necesidad de perfiles que no sean tan genéricos y se actualicen, ajustándolos mejor a las exigencias del puesto. Se debería huir de concursos que premian a los acumuladores de méritos y/o años.

Estamos lejos de la volatilidad en el puesto que se produce en los Estados Unidos (EUA), donde se hace necesaria una mayor estabilidad (Hernon, 2011), pero estos puestos tampoco deberían ser necesariamente hasta la jubilación. El impulso y dinamismo que requieren, así como la factura personal que hacen pagar, parecen incompatibles con grandes permanencias. No es el objeto concreto de este estudio, pero se debería establecer también una revisión de la idoneidad en el tiempo.

$\mathrm{Si}$ atendemos al plano estrictamente administrativo, el problema sí que ha tenido una amplia repercusión en la investigación. Así, Cuadrado (2009: 2) indica que: 
Pese a los intentos de reforma y modernización de la Administración Pública española, sigue siendo una asignatura pendiente implantar una verdadera política de recursos humanos en el escalón directivo de la estructura organizativa. Ello es debido a que la provisión de los puestos responde más a criterios partidistas y clientelares que a criterios estrictamente profesionales.

Aunque la Ley 6/1997, de 14 de abril, de Organización y funcionamiento de la Administración General del Estado (LOFAGE) limita en su art. 6 la categoría de órgano directivo hasta el nivel de subdirectores generales como mínimo, el advenimiento del Estatuto Básico del Empleado Público (EBEP) (Ley 7/2007, de 12 de abril) debería suponer la asimilación de estos puestos de jefe de servicio a la figura del directivo público, dada su autonomía de actuación, el tamaño y el alcance transversal de estas unidades. Por ello, se debería modificar su aspecto y forma de cubrirlo, siguiendo además a la propia LOFAGE, que indica en su artículo 6.10 que "Los titulares de los órganos directivos son nombrados atendiendo a criterios de competencia profesional y experiencia".

Desafortunadamente, los desarrollos y regulaciones que esto implicaría aún no se han producido y parece incluso lejana esa posibilidad. En el caso concreto de los ámbitos bibliotecarios, lo único con lo que prácticamente contamos son los temarios del Cuerpo Facultativo de Bibliotecas, en sus diversas denominaciones según la administración convocante, que no sirven evidentemente para este fin, aunque se está trabajando en su actualización al estilo de lo ya realizado en Francia. Además, podemos echar mano del perfil de "Director de biblioteca" del completo estudio sobre la definición de puestos profesionales del Consejo de Cooperación Bibliotecaria (CCB) (Tejada y Martínez, 2013: 9).

Resulta interesante recuperar la Propuesta sobre política de bibliotecas y de archivos a los partidos políticos para su incorporación en los programas electorales y de gobierno de la Federación Española de Sociedades de Archivística, Biblioteconomía, Documentación y Museística (FESABID, 2007). En ella se solicita "un estatuto marco profesional de los bibliotecarios", en el que se determinen escalas, funciones y competencias, junto con un "modelo homogéneo de selección y de promoción". Igualmente, se pide que "el nombramiento de los máximos responsables de las bibliotecas y los sistemas bibliotecarios se base en la experiencia, el conocimiento y el prestigio de los candidatos".

\section{OBJETIVOS Y METODOLOGÍA}

La finalidad principal de este trabajo es el acercamiento a la definición del perfil de directivo de biblioteca universitaria en España, previsiblemente extrapolable con mínimas modificaciones a la realidad internacional.

Con ello se debe comenzar a completar un vacío detectado en la literatura profesional sobre bibliotecas, además de abrir el camino para trabajos similares que sería deseable repetir en otros puestos de responsabilidad con carácter técnico, tanto en la universidad como en el resto de las administraciones públicas. Se pretende así reforzar el acceso según el mérito de las personas que concursan a puestos de libre designación, asegurando un mínimo de competencias necesarias que permitan un eficaz desempeño en el puesto.

Este objetivo general se complementa con una serie de objetivos específicos y consecuentes con el mismo, como puede ser la difusión de la necesidad de una mayor profesionalización en el acceso al cargo; sentar las bases para un futuro desarrollo del tema; o proporcionar la base para el desarrollo de herramientas de utilidad para la selección de los directores. 
La consecución de estos objetivos parte de una metodología analítica y lógico deductiva, que comenzó con la búsqueda exhaustiva en fuentes bibliográficas. Además, se realizó una búsqueda sistemática de ofertas de empleo de director o decano (dean) de biblioteca universitaria, incidiendo especialmente en la ALAJobList o página de ofertas de la American Library Association.

Se debe señalar que los resultados se centraban en bibliotecas norteamericanas (EUA y Canadá) y británicas, y se restringió a aquellas vigentes o recientemente aparecidas (septiembre a diciembre de 2015). Esto es debido a los diferentes tipos de acceso a estos puestos en otros países, así como a la realización de la búsqueda en inglés, pero también fue un resultado deseado. Se pretendían conseguir ofertas actualizadas de las que extraer los requisitos habituales para el puesto en un entorno muy avanzado bibliotecariamente y que, al no estar sujeto a la contratación por medio de los mecanismos de la función pública, asegurase la presencia de los contenidos buscados.

Con la información obtenida se procedió al análisis inductivo, reflexivo y crítico, generando en el plano teórico las cualidades y conocimientos que según los diversos autores debe tener un gestor o directivo de bibliotecas. De la misma manera, la comparación de las ofertas permitió la extracción de unas pautas generales de los requisitos demandados en las mismas a los participantes. A ello se añadió la experiencia y conocimientos personales sobre la realidad de los puestos a debate en este trabajo.

De esta forma se han podido deducir una serie de competencias para la elaboración de un perfil-tipo de director de biblioteca universitaria. La circunscripción a la tipología de bibliotecas universitarias se debe a que fuera de ese ámbito de la administración los puestos sujetos al procedimiento de libre designación son mucho más escasos.

\section{EL DIRECTOR/A DE BIBLIOTECA COMO GESTOR PÚBLICO}

Como hemos visto, la dirección de la biblioteca reúne una doble naturaleza como bibliotecario y como gestor público. En la práctica, esto también supone añadir que se trata de un funcionario. Raramente se produce en España la ocupación de estos puestos por personal eventual, aunque hay casos conocidos. En las bibliotecas universitarias el gestor frecuentemente tiene un responsable académico por encima, normalmente sin ningún tipo de conocimiento técnico, a modo de supuesta supervisión.

Ya nos hemos referido a la concepción del director de una biblioteca de gran tamaño como un gestor encuadrable en lo que se podría concebir como directivo público. De hecho, el director o directora de una gran biblioteca universitaria española tiene a su cargo más personal y presupuesto que buena parte de los directores generales. Esto nos hace reflexionar, en este primer apartado, sobre aspectos y cualidades de carácter general del mismo como funcionario público.

Es sabido que la OCDE es uno de los mayores impulsores de la creación de la figura del directivo público, de la función pública profesional (OCDE, 2008), en parte por la necesidad de introducir "cierto grado de flexibilidad en la selección y en las condiciones de trabajo de los directivos públicos" en selección, remuneraciones, condiciones laborales, etc., (Blanco, 2010: 183). Sin embargo, esto en España debería tener ciertas limitaciones puesto que cuenta con un "sistema de carrera" que reserva ese tipo de puestos a funcionarios.

Esto parece positivo, pues asegura cierta estabilidad en la administración. También que la persona tenga unos conocimientos y capacidad de esfuerzo y estudio demostrada por la oposición (otro tema sería hablar de los empleados públicos que han accedido a estos puestos 
sin pasar por este tipo de procesos o con ellos preparados ad hoc) y una preparación previa otorgada por la propia carrera administrativa, los años de experiencia, cursos y aprendizaje en general. Como indica Blanco (2010: 187), esta circunstancia además ayuda a la "existencia de una cultura común y de unos mismos valores públicos", propios de los sistemas de carrera.

Por ello, la presión ejercida por la OCDE para la apertura de estos puestos al sector privado parece un error. Es conveniente que el gestor público cuente con unos conocimientos y capacidades que le permitan una administración más eficaz y eficiente, pero esto no es incompatible con su condición de funcionario público. Sin embargo, la idea de servicio público y el conocimiento de las peculiaridades de estas administraciones sí están fuera del bagaje de un gestor privado. También puede aprenderlo, pero tras un tiempo de graves disfunciones en su cometido. Eso parece tenerlo claro el redactor del citado EBEP, que casi asegura la condición de funcionario del empleado público.

No obstante, todo ello no deja de indicar la necesidad de que este gestor público reciba unas herramientas formativas con las que ahora mismo a menudo no cuenta y que son prácticamente ignoradas en su proceso selectivo. De ahí la redacción de este trabajo y la necesidad de la existencia de instrumentos de formación (y acreditación) de esos conocimientos, en la forma de escuelas de gestión pública, cualquiera que sea el nombre que se le quiera otorgar. Así, se expresa el anhelo de que en un país como el nuestro, con buena parte de su legislación y del sistema administrativo y funcionarial inspirado en el modelo francés, se adopte también algo semejante a la ENA (Escuela Nacional de Administración). El desarrollo de este propio artículo evidencia la necesidad de que esa formación tenga una faceta doble: "una formación genérica pluridisciplinaria y básica, a la que se añadiría con carácter complementario una formación especializada", propia de entidades de la "administración educativa y cultural" que nos ocupa (Canales, 2015: 80).

Como decimos, el EBEP es la primera norma que adopta la idea del directivo público, aunque de forma muy abierta, para que sea adaptada a las necesidades de las distintas administraciones (Blanco, 2010: 192-193). Desafortunadamente, el desarrollo reglamentario posterior brilla por su ausencia, pero el propio texto regula algunos aspectos como la reserva de estos puestos a funcionarios en la práctica: "en todo caso, el ejercicio de las funciones que impliquen la participación directa o indirecta en el ejercicio de las potestades públicas o en la salvaguardia de los intereses generales del Estado y de las Administraciones públicas corresponden exclusivamente a los funcionarios públicos, en los términos que en la ley de desarrollo de cada Administración se establezca (Artículo 9.2)". (Blanco, 2010: 192-193).

Añade la propia autora otros elementos de interés de esta regulación, como que "su designación atenderá a principios de mérito y capacidad y a criterios de idoneidad, y se llevará a cabo mediante procedimientos que garanticen la publicidad y concurrencia (Artículo 13.2)." Sobre lo primero, es una de las causas que han impulsado este trabajo; y sobre la publicidad y concurrencia, al margen de los anuncios en boletines oficiales y las consiguientes herramientas de alerta, resulta significativo y esperanzador un caso eminentemente bibliotecario. La reciente Ley 1/2015, de 24 de marzo, reguladora de la Biblioteca Nacional de España, ha promovido que la selección de la dirección de la misma se realice "atendiendo a criterios de competencia profesional y experiencia en relación a los fines y objetivos de la Biblioteca Nacional de España." (Artículo 9.1). Para ello "se acudirá a un sistema de preselección que garantice la publicidad y concurrencia, y que asegure la participación del Real Patronato, con el asesoramiento de profesionales del ámbito bibliotecario y documental. Su designación atenderá a los principios de mérito, capacidad e idoneidad." Todo ello teniendo una categoría de Director General. Así ha ocurrido realmente, en una convocatoria en la que además se ha asegurado el anonimato de los candidatos. 
Siendo, como hemos visto, el aspirante a uno de estos puestos funcionario público, lo habitual sobre su procedencia es que sea de la propia institución, pero esto tiene tantas ventajas como inconvenientes. Evidentemente, su conocimiento de la situación real de la institución y, aún más importante, de las relaciones entre las personas y equipos, resulta muy beneficiosa de cara al éxito inmediato. Por el contrario, un candidato interno puede verse condicionado por las relaciones personales establecidas a lo largo de los años. El cambio de colega a responsable conlleva también dificultades y una carga psicológica significativa al pasar a pedir tareas y responsabilidades a los anteriores compañeros (Newcombe \& Donovan, 2013), pero una persona externa puede y suele ser vista como un intruso en los cotos cerrados de las administraciones españolas.

De todo lo anterior se trasluce la necesidad de que la selección asegure el mérito de los candidatos. Sin esta vocación meritocrática, este trabajo ni siquiera se hubiese planteado, pues lo que se pretende, como hemos visto, es que el proceso se ajuste lo más posible a los principios constitucionales de igualdad, mérito y capacidad, y para ello se deben perfilar las herramientas necesarias.

La importancia de la meritocracia en el acceso a los puestos de responsabilidad en la administración pública

La meritocracia en el proceso de acceso y selección de los funcionarios y trabajadores públicos es uno de los fundamentos de la administración pública weberiana. Con ello se asegura su profesionalidad, que deviene en un mejor funcionamiento de la burocracia, así como en una independencia de los poderes políticos, fundamental como barrera frente a la corrupción.

Esto ha sido refrendado incluso por estudios empíricos mediante encuestas, como el informe Organizing Leviathan: Politicians, bureaucrats and Government (Dahlström y Lapuente, 2014). En el mismo, España aparece en una situación relativamente buena, aunque con amplio margen de mejora para acercarnos a los modelos neozelandés, australiano o de los países nórdicos, donde parece que el mérito y la capacidad son verdaderamente los parámetros para la selección de los funcionarios. Como resultado, gozan de una mayor eficiencia de la administración, así como de unos niveles más bajos de corrupción.

La salvaguarda de la meritocracia supone una apuesta de largo recorrido por el aseguramiento de la calidad de los servicios públicos. Canadá es un ejemplo de que el debilitamiento de la meritocracia en el acceso al servicio público deviene en un empobrecimiento del mismo a todos los niveles, incluida la eficacia y eficiencia que se buscaba (Sánchez, 2007). Y es que si no se realiza una selección y designación basándose en el mérito y capacidad, entramos de forma inevitable en lo que Corrales Melgarejo (2011), un juez peruano, denomina padrinocracia. De esta forma tan ilustrativa define la dicotomía que se presenta entre la selección de acuerdo al mérito y el enchufismo, que suele derivar y asociarse al clientelismo.

Además, la meritocracia es uno de los fundamentos de cualquier reforma o modernización administrativa, pues requiere de una función pública estable y profesional, en el que el acceso y la promoción se realicen en función del mérito y capacidad de los solicitantes (Martínez, 2013: 172). En ello, se defiende que se debe aplicar la Nueva Gestión Pública (NGP) sin que esto implique la destrucción del principio de la meritocracia (Abril et al., 2013). Eso sí, esta meritocracia debe ser revisada y actualizada. No sirven en un entorno cambiante como el actual los mismos métodos y criterios de antes, sino que la meritocracia debe estar ligada al aprendizaje, al esfuerzo y a los resultados alcanzados (Longo, 2010). Así se evitaría la paralización de la ambición del funcionario ante un sistema de carrera poco motivador. 


\subsection{Principios generales de los directivos y funcionarios públicos}

Este funcionario del que estamos hablando, en nuestro caso bibliotecario, que puede optar al puesto de dirección, compartirá con otros directivos públicos, y en buena medida con los gestores privados, una serie de principios generales. El conocido Informe Nolan indica siete principios de la Vida Pública, con los que la persona que estamos perfilando debe contar (Canales, 2012: 23):

a) Desinterés. Se debe orientar la gestión hacia la sociedad, no pensando en primer lugar en los intereses propios y del equipo.

b) Integridad. La relación con proveedores, editores, etc. no debe implicar un vínculo financiero ni de otro tipo con los mismos.

c) Objetividad. Aplicable a la gestión de recursos humanos, a la contratación de productos y servicios o la adquisición de materiales bibliográficos.

d) Responsabilidad. Los cargos públicos deben estar dispuestos a su propia evaluación, tanto en su desempeño como sobre la permanencia de su idoneidad, y a rendir cuentas de su gestión.

e) Transparencia. La toma y justificación de las decisiones debe ser un proceso lo más claro posible, tanto de cara a los subordinados como a los usuarios y comunidad universitaria.

f) Honestidad. Se debe actuar siempre de forma que sus decisiones, en particular las que impliquen un gasto, no puedan ser puestas en duda más allá de la estricta legalidad de las mismas.

g) Liderazgo. Se convierte en aspecto fundamental del gestor público, tanto en lo que se refiere a su persona, como al fomento del mismo dentro de los equipos.

Varios de estos principios nos hablan de la ética del directivo público. Al respecto, la profesión bibliotecaria cuenta con varios códigos éticos y deontológicos, de los que los más conocidos en nuestro ámbito son el Código de Ética de la IFLA para bibliotecarios y otros trabajadores de la información y el Código Ético para bibliotecarios y profesionales de la información en España. García y Sutherland (2001) hablan de la ética en su relación con el gestor político, señalando que, aunque sea necesaria la flexibilidad y la mano izquierda, no se puede renunciar a la misma cuando sea cuestionada en contrataciones o gestión de las colecciones y servicios.

La ética, debido a los múltiples casos de corrupción surgidos en España en los últimos años, ha pasado a ser tomada en consideración en el ámbito de la Función Pública, completando y superando al principio de legalidad de las actuaciones de los funcionarios (Irurzun, 2010). Difícil, no obstante, resulta valorarla, más allá de los requisitos básicos, como el no haber sido separado del servicio o inhabilitado.

El liderazgo como cualidad y competencia clave en los puestos directivos

El último punto del Informe Nolan habla del liderazgo como principio esencial del gestor público. De todas las cualidades que debe tener el directivo, consideramos que la capacidad de liderazgo es la clave. Así lo demuestra la muy abundante bibliografía internacional sobre el tema hasta el punto de a veces confundir, erróneamente, la figura del líder con la del director o gestor.

El liderazgo es importante en cualquier organización privada, pero en las administraciones públicas resulta fundamental, pues debe gestionar equipos sin herramientas coercitivas; incluso más allá, es la cualidad definitoria para un gestor que quiera llevar su organización hacia nuevas metas, incluyendo en este caso la modernización administrativa o la proyección futura de las bibliotecas. Como ejemplo, el mencionado programa de doctorado 
norteamericano (Hernon \& Schwartz, 2008) se centraba en el liderazgo gerencial como clave para la gestión de instituciones documentales.

A estas alturas es evidente que el liderazgo no sólo es imprescindible para las jefaturas, sino que resulta importante en todos los niveles de la biblioteca. Sería necesario detectarlo incluso desde el primer proceso selectivo (DeLong, 2009: 455), algo, no nos engañemos, casi imposible con nuestro sistema de oposiciones.

Resulta incluso "urgente" (Maloney, Antelman, Arlitsch \& Butler, 2010) que en las actuales circunstancias los directivos fomenten también el liderazgo en su organización, siendo necesario para el futuro de la biblioteca y su capacidad de adaptación, cambio y desarrollo. Frecuentemente las organizaciones fallan en ello, siendo instituciones muy rígidas y jerarquizadas que ahogan el desarrollo de líderes. Eso se produce por la propia naturaleza weberiana de las estructuras burocráticas, pero también, desgraciadamente y a veces, por simple temor. El directivo mediocre teme que el afloramiento de líderes le deje en evidencia (Maloney et al., 2010).

Contrariamente a este punto de vista, Carpenter (2012) ensalza la labor realizada por diferentes directores de bibliotecas de la Association of Research Libraries (ARL) para fomentar el liderazgo. Particularmente, el liderazgo orientado al emprendimiento, como un recurso fundamental en nuestras bibliotecas en esta época de crisis, y como vía para la detección y aprovechamiento de oportunidades.

Los directores de bibliotecas deberían contar con esta capacidad de emprendimiento, esta facilidad para la búsqueda de oportunidades, liderando la misma y permitiendo y aceptando los fracasos (Carpenter, 2012). Al tiempo, debemos tener en consideración que los líderes que se formen en el equipo puede que luego no sean buenos gestores, pues ese liderazgo, cuando se pasa a puestos gerenciales, muchas veces se ve más constreñido que liberado (White, 1997).

El liderazgo implica "tener una visión clara de hacia dónde se dirige la organización, ser capaz de comunicar esto y motivar al personal para que integre sus objetivos personales con los de la organización", ya que el directivo/líder necesita contar con este personal y organizarlo adecuadamente para obtener los resultados que se buscan (Weingand, 1999).

Para ello, el liderazgo requiere del uso y, en su caso, conocimiento, aunque sea intuitivo, de la inteligencia emocional, hasta el punto de ser considerada la condición sine qua non del mismo (Goleman, Boyatzis \& McKee, 2002: 5). La inteligencia emocional, de acuerdo a estos autores, consiste en 18 rasgos que, si bien son innatos, pueden ser mejorados o entrenados; y de los que los grandes líderes desarrollan unos 12. Al respecto, Kreitz (2009) elaboró una lista de los rasgos que deben caracterizar al liderazgo dentro de las bibliotecas, de acuerdo a los diferentes niveles de dirección, que no traemos aquí por su notable extensión.

El liderazgo ha sido trabajado y tratado en múltiples cursos, artículos y libros, pero muchos de ellos no dejan de ser obras de autoayuda, y la aproximación hacia el liderazgo es a menudo limitada (Hernon \& Schwartz, 2011). El fomento del liderazgo debería estar presente en los planes de estudio de Documentación y, aún más, de las escuelas de administración pública.

Al respecto, la Fundación Bill y Melinda Gates publicó en 2015 el informe Cultivating Global Library Leadership: a Review of Leadership Training Programs for Librarians Worldwide (Arabella Advisors, 2015). En él se destaca la escasez de programas de formación de líderes bibliotecarios, sobre todo fuera de los países desarrollados, y la importancia de los mismos en un mundo cambiante, aunque también se señala la "falta de consenso sobre qué habilidades debe tener un líder" (FGSR, 2015). 


\section{EL DIRECTOR DE BIBLIOTECA COMO BIBLIOTECARIO Y COMO GESTOR DE BIBLIOTECAS.}

Además de las competencias y habilidades de carácter general vistas hasta ahora, las competencias que debe atesorar como bibliotecario y gestor de la biblioteca están especialmente influidas por la evolución acelerada de la profesión y de su entorno a la que hemos hecho referencia. Así, la transformación de las bibliotecas y de sus servicios ha suscitado la necesidad de nuevos perfiles profesionales con conocimientos que no siempre se encuadran en los tradicionales estudios de documentación. Esto ha provocado que incluso en mercados laborales menos regulados como el norteamericano se comiencen a contratar personas de perfiles profesionales ajenos a la biblioteconomía o sin estudios específicos de la materia (Grimes \& Grimes, 2008).

Si nos dirigimos a un trabajo reciente sobre el tema, el estudio Prospectiva 2020 (GalloLeón, et al., 2014) dedicaba una de sus 10 tendencias de futuro para las bibliotecas al personal del mismo, indicando que "los profesionales deberán tener perfiles flexibles y cambiantes y las bibliotecas necesitarán personal con conocimientos diversos".

De esta forma, el documento señala que las bibliotecas, debido a su entorno inestable, necesitan profesionales de una tipología nueva, polivalentes $\mathrm{y}$, por tanto, capaces de desenvolverse en muy diversas áreas:

"Las TIC y los cambios sociales que han venido con ellas han cambiado la profesión de forma ostensible en los últimos años. (...) El personal que precisan las bibliotecas, además de las destrezas tradicionales, deberá tener o ser capaz de adquirir competencias y habilidades variadas, que no siempre serán tecnológicas: sobre gestión y organización (incluyendo economía o estadística), capacidades educativas en alfabetización informacional, habilidades de negociación, conocimientos sobre comunicación (donde se incluye las tecnologías de la información) y otros conocimientos (propiedad intelectual y derecho, idiomas, sociología, pedagogía, etc.)" (Gallo-León, et al., 2014: 49).

Muchas de estas capacidades serán empleadas tanto en la organización como de forma vertical en el trato con los responsables superiores, de cariz político aunque algunos sean cargos académicos. Recordemos que la prosperidad de la biblioteca dependerá de la voluntad política de estos cargos. Por ello, los bibliotecarios académicos deben estar preparados para su relación con el político, como si de una biblioteca pública se tratase. Esto requiere de bastantes de las cualidades y conocimientos que se expresan en estos apartados, como podemos comprobar en la obra de García \& Sutherland (2001).

\subsection{Visiones sobre las competencias del bibliotecario en el actual entorno de cambio}

Además de las competencias profesionales, el bibliotecario académico necesita un amplio conjunto de competencias personales y transferibles para poder gestionar el entorno de cambios en el que trabaja (Joao, Extremeño y Firmino, 2012: 96). Empezando por las propuestas de la influyente Special Library Association (SLA), esta definía las competencias de un profesional de la información en el siglo XXI (Abels, Jones, Latham, Magnoni, \& Marshall, 2003), diferenciando entre competencias profesionales y competencias personales.

Entre las profesionales, se encuentran: la gestión de recursos de información, gestión de servicios de información, gestión de organizaciones o instituciones de información y la capacidad de aplicar las herramientas y la tecnología de la información. Mientras, entre las 
personales podemos destacar la búsqueda de desafíos, la visión, la capacidad de generar alianzas, la innovación, la capacidad de planificación en general, etc.

Siguiendo en un plano internacional, pero con un carácter transnacional, europeo y fruto de un trabajo colaborativo, el Euroreferencial es un trabajo realizado por el European Council of Information Associations (ECIA) hace ya algunos años (2004), que recogía las competencias y aptitudes propias de los profesionales de la información y la documentación en un marco europeo.

Desde un punto de vista subjetivo, y a pesar del tiempo transcurrido, resulta bastante vigente la visión de Anglada (1993), justo en el inicio de la anterior gran crisis económica que también lo estaba agitando todo, sobre el futuro inmediato de los perfiles bibliotecarios. En ella ya se habla de orientación al mercado y al usuario, no al producto, experticia en TIC, capacidad de trabajo en equipo e interdisciplinar, etc. Algo posterior, Moreiro (1999) recogía requisitos exigidos para acceder al trabajo entre los que resulta destacable la presencia, junto con conocimientos técnicos básicos, de la exigencia de idiomas, la facilidad para las relaciones públicas o la informática.

Joao et al., (2012: 100) establecen que, en el marco de los cambios de la biblioteca universitaria en el entorno actual y ante el nuevo paradigma de la biblioteca como centro de aprendizaje y apoyo a la investigación, los bibliotecarios adoptan nuevos roles que, principalmente, se centran en su función como formador en alfabetización informacional y facilitador del aprendizaje; y como gestor del conocimiento y editor de contenidos, lo cual se desglosa en una serie de papeles como: gestor de colecciones, prestador de servicios y consultor de información, gestor del conocimiento, etc.

\subsection{Revisión de las competencias del gestor de biblioteca según las fuentes profesionales}

En lo referente a competencias más específicas del gestor bibliotecario, el Middle Management Institute de la SLA fijaba cinco materias como fundamentales para el directivo intermedio, prácticamente asimilable al superior: tecnología de la información y aplicaciones; recursos humanos; marketing y relaciones públicas; habilidades de gestión y administración; y herramientas analíticas (Taylor \& Renzulli, 1997).

Más influyente aún es el punto de vista de la ALA (American Library Association, 2015), que en su web publica una guía para directores o futuros directores de biblioteca, en la que, habla de sus requisitos habituales divididos en tres ámbitos: formación, habilidades y experiencia.

Younger (2008), por su parte, no habla de competencias sino de habilidades de dirección, agrupándolas en tres categorías principales:

a) Habilidades de gestión práctica (recursos humanos, presupuesto, las operaciones, administración y gestión de los recursos).

b) Habilidades relacionadas con la promoción y la persuasión (negociación, hablar en público, habilidades de escritura, búsqueda de financiación y comprensión de la psicología y la política interna).

c) Creatividad e innovación, sobre lo que también insistía el citado Anglada (1993).

Además, existen dos interesantes estudios que se han basado en el trabajo con expertos y directores de bibliotecas para intentar especificar las habilidades/competencias más importantes para este tipo de puestos. El primero (Jordan, 2011) consistió en la identificación de las competencias en la bibliografía y de esta manera crear unas tablas que fueron sometidas a un estudio Delphi con tres rondas entre los directores de las consideradas 100 mejores bibliotecas públicas norteamericanas. Para seleccionar las mismas se basó en los 
Hennen's Annual Public Library Ratings, que especifican las 10 mejores bibliotecas del año en diferentes categorías.

Aún orientado, como vemos, a bibliotecas públicas, la mayoría de las cuales tienen otras exigencias de personal, presupuesto y colecciones, creemos que es un trabajo suficientemente significativo para ser tenido en cuenta. Sorprendentemente, en la primera ronda del estudio ya se eliminó como competencia importante la Inteligencia Emocional, muy tenida en cuenta de forma general, pero aun así la tabla final proporciona unos resultados que son muy significativos, incluyendo el entusiasmo, liderazgo, capacidad de delegación, responsabilidad, planificación, integridad, toma de riesgos, credibilidad, gestión de recursos, creatividad, orientación al cliente, habilidades interpersonales y de comunicación, visión, comprensión política, madurez, capacidad de resolución de problemas y habilidades de promoción.

En el segundo estudio que mencionamos (Chow \& Rich, 2012), se hicieron 114 entrevistas a directivos de biblioteca, pero con la limitación de que todos procedían del estado de Carolina del Norte. En el mismo se diferenciaba entre "cualidades" y "tareas más comunes". Entre las primeras, la empatía fue la que se señaló más frecuentemente $(41 \%)$, seguida de la visión (21\%), flexibilidad (14\%) y habilidades comunicativas (14\%). La capacidad de delegación (5\%) y la integridad (5\%) también fueron citadas repetidamente. Sobre las tareas principales, la más mencionada en las entrevistas fue el servicio a los usuarios (22\%), seguido de la gestión presupuestaria (15\%) y la gestión de la colección.

El trabajo más importante, por cercanía y profundidad, es el realizado por el Grupo de Trabajo sobre Perfiles Profesionales del CCB (Tejada y Martínez, 2013). Basado en las competencias recogidas en el Euroreferencial, recoge las específicas para el perfil de director en una completa ficha que incluye funciones, competencias profesionales y competencias personales (aptitudes).

De todos estos conocimientos y habilidades recogidos hay tres que necesitarían un mayor desarrollo. Por una parte, si la formación es actualmente uno de los ejes transversales de la profesión bibliotecaria, esto se convierte en acuciante para las bibliotecas universitarias, donde no se trata sólo de poseer capacidades educativas y formativas, sino de ampliar estos conocimientos al propio hecho del aprendizaje.

Por otra parte, las TIC han sido el motor del cambio de paradigma que han vivido las bibliotecas universitarias. Este cambio de modelo no viene dado directamente por la tecnología, sino por el cambio de relación entre la biblioteca y el usuario que esta tecnología, entre otros factores, ha desencadenado. Es el agente provocador del cambio social y, por tanto, bibliotecario. El conocimiento de las tecnologías deberá habilitar al bibliotecario para el discernimiento de las más adecuadas para su trabajo y para el servicio de la biblioteca.

Además, el bibliotecario deberá estar capacitado para la autopromoción: "Para poder afrontar el futuro desde una perspectiva influyente, el bibliotecario (en particular el especializado) deberá venderse, dejar claro quién es y qué puede hacer por sus usuarios, sin dar nada por sentado, tanto dentro como fuera de la institución que lo aloja" ( et al., 2014).

\subsection{Análisis de las ofertas de trabajo consultadas}

Tras el análisis de las diversas ofertas de trabajo podemos obtener una idea aproximada de las exigencias medias reales para el puesto de dirección en una biblioteca académica. Como conclusión previa general, podemos ver que los requisitos son similares, aunque a veces cambie sutilmente su denominación. Además, el nivel de exigencia es muy variable en función del prestigio de la institución ofertante. 
Todas las ofertas en los EUA solicitaban un mínimo de titulación de Máster en Library Science (Biblioteconomía) acreditado por la ALA, salvo condiciones de carreras personales excepcionales. La práctica totalidad preferían que el título fuese un doctorado $(\mathrm{PhD})$, algo interesante, pues apenas se valora en los concursos españoles o no se hace en absoluto.

También existía una gran coincidencia en la necesidad de una experiencia demostrable en bibliotecas académicas, algunas veces en puestos de gestión. Normalmente se elevaba a 10 años, aunque otras veces se restringía a 5 o no se especificaba la cuantía.

Dentro de las aptitudes personales, se repetía el liderazgo, así como la capacidad de comunicación. Igualmente se valoraba reiterativamente la capacidad de trabajo en equipo, a la par que la capacidad de trabajar y tomar decisiones de forma autónoma.

$\mathrm{La}$ facilidad en las relaciones interpersonales tiene un gran protagonismo. Varias solicitaban la capacidad de formar consensos y trabajar en entornos diversos, y la mitad hablaban del fomento de la diversidad y la integración.

En lo que se refiere a la gestión pura, se habla frecuentemente de las capacidades de organización y supervisión. De la misma forma se insiste en la experiencia y conocimientos de técnicas de planificación, especialmente contar con una visión clara del desarrollo de las bibliotecas, lo cual vemos refrendado en los dos siguientes apartados.

Mayoritariamente se habla de conocimientos sobre las TIC e informáticos en general, poniéndolos en relación normalmente con la capacidad de evaluación de tecnologías emergentes y nuevas aplicaciones: su utilidad y posible incorporación.

En cuanto a los conocimientos puramente bibliotecarios, se valora estar al día, a veces especificándose aspectos concretos (gestión de la colección, referencia, etc.), pero casi siempre con una orientación hacia el futuro. Esto es, se valora la capacidad de análisis y prospectiva de tendencias en los servicios bibliotecarios. Se repiten cuatro veces las referencias a la alfabetización informacional.

Las dos ofertas que pueden ser consideradas europeas (aunque una corresponda al Tribunal de La Haya) añaden, como es lógico, la capacidad de trabajar en un ambiente internacional y valoran el conocimiento de varios idiomas, además del obligatorio inglés.

Como aspecto curioso, solo una oferta habla de la orientación al servicio público. Resulta sorprendente, desde nuestro punto de vista, que tres de ellas valoren la pertenencia y actividad en organizaciones nacionales e internacionales de bibliotecarios. Por fin, resulta anecdótico, pero muy significativo, que en una oferta se especifique que hay que subir 20 escalones diarios, y en otra ser capaz de desplazarse de un edifico a otro del campus.

\section{PROPUESTA DE PERFIL DE DIRECCIÓN DE BIBLIOTECA UNIVERSITARIA BASADA EN COMPETENCIAS}

Como hemos visto en los apartados anteriores, el perfil de dirección para una biblioteca va a tener una serie de componentes que se hacen comunes y reiterativos en los diferentes acercamientos. Se deben conjuntar una serie de cualidades innatas, mejoradas con el estudio y el trabajo, más una serie de conocimientos. Esto hará que reúna las cualidades de cualquier gestor o directivo público con una serie de conocimientos técnicos, necesarios para su desempeño y para que tenga la ineludible visión y capacidad de toma de decisiones. Evidentemente, es muy complicado reunir todas estas competencias en una sola persona, y las circunstancias del puesto harían cambiar el nivel de exigencia sobre ellas.

A lo largo del trabajo hemos visto que los autores han utilizado, muchas veces como sinónimos, diferentes términos: habilidades, conocimientos, rasgos, capacidades, etc. Como 
en realidad no son sinónimos, aquí aplicaremos, ligeramente modificada, la definición dada en el estudio Prospectiva 2020 (Gallo-León, et al. 2014: 49), donde se definen las competencias como un conjunto de actitudes, habilidades, valores y conocimientos que han de tener los bibliotecarios para realizar su trabajo de forma efectiva y contribuir positivamente en sus organizaciones, usuarios y profesión, y que pueden ser desarrolladas, entrenadas y mejoradas mediante el aprendizaje.

Las competencias se pueden agrupar en cuatro familias o clases: personales y emocionales; gerenciales; tecnológicas; y bibliotecarias. A ellas se añadirían una serie de requisitos para el ejercicio del puesto, algunos de ellos ineludibles por ley, que se refieren a la cualificación profesional y la experiencia. Las competencias son ordenadas según su importancia de mayor a menor, de acuerdo a la reiteración de su aparición en las fuentes consultadas, ligeramente modificadas según el criterio propio. Asimismo, las denominaciones han sido adaptadas para refundir competencias que aparecían bajo términos sinónimos. Finalmente, se debe indicar que las limitaciones espaciales propias de un trabajo como este impiden detenerse en la descripción meticulosa de cada competencia.

Propuesta de competencias para el perfil de director de biblioteca universitaria Fuente: Tablas 1-5 elaboración propia

Tabla 1: Competencias personales y emocionales

\begin{tabular}{|c|c|}
\hline Denominación de la competencia & Forma de valoración \\
\hline $\begin{array}{l}\text { 1. Liderazgo } \\
\text { 1.1. Ética } \\
\text { 1.2. Honestidad } \\
\text { 1.3. Deontología profesional } \\
\text { 1.4. Integridad } \\
\text { Comunicación: } \\
\text { 2.1. Capacidad de redacción y realización de informes } \\
\text { 2.2. Exposición y presentación de contenidos (escrita y oral) } \\
\text { 2.3. Capacidad de escucha } \\
\text { 2.4. Capacidad de diálogo } \\
\text { 2.5. Capacidad de negociación } \\
\text { Relaciones interpersonales } \\
\text { 3.1. Inteligencia emocional en general } \\
\text { 3.2. Trabajo en equipo, creación de alianzas } \\
\text { 3.3. Asertividad } \\
\text { 3.4. Reconocimiento de los éxitos } \\
\text { Cualidades personales y mentalidad } \\
\text { 4.1. Voluntad de servicio y orientación al usuario } \\
\text { 4.2. Pensamiento analítico } \\
\text { 4.3. Iniciativa } \\
\text { 4.4. Criterio independiente } \\
\text { 4.5. Entusiasmo } \\
\text { 4.6. Madurez } \\
\text { 4.7. Coraje y toma de riesgos } \\
\text { 4.8. Tenacidad } \\
\text { 4.9. Positividad } \\
\text { 4.10. Credibilidad }\end{array}$ & $\begin{array}{l}\text { Formación recibida y } \\
\text { certificada: cursos y paso por } \\
\text { escuela de dirección pública. } \\
\text { - } \quad \text { Entrevista } \\
\text { - Indagación en el entorno }\end{array}$ \\
\hline
\end{tabular}


Tabla 2: Competencias gerenciales

\begin{tabular}{|c|c|}
\hline Denominación de la competencia & Forma de valoración \\
\hline $\begin{array}{l}\text { 1. Planificación } \\
\text { 1.1. Visión } \\
\text { 1.2. Flexibilidad } \\
\text { 1.3. Planificación estratégica } \\
\text { 1.4. Gestión de calidad y evaluación } \\
\text { 1.5. Organización y coordinación } \\
\text { 1.6. Desarrollo de proyectos } \\
\text { 1.7. Marketing y capacidad de autopromoción } \\
\text { 1.8. Orientación a la innovación } \\
\text { 1.9. Gestión y promoción del liderazgo } \\
\text { 1.10. Capacidad de delegación } \\
\text { Conocimientos de gestión pública } \\
\text { 2.1. Gestión de recursos humanos } \\
\text { 2.2. Gestión financiera } \\
\text { 2.3. Presupuestación y valoración de los costes de } \\
\text { actividades y proyectos } \\
\text { 2.4. Procedimiento administrativo } \\
\text { 2.5. Conocimiento de la estructura universitaria }\end{array}$ & $\begin{array}{l}\text { - Formación recibida y certificada: } \\
\text { cursos y paso por una escuela de } \\
\text { dirección pública. } \\
\text { - Experiencia }\end{array}$ \\
\hline
\end{tabular}

Tabla 3: Competencias tecnológicas

\begin{tabular}{|c|c|}
\hline Denominación de la competencia & Forma de valoración \\
\hline $\begin{array}{l}\text { 1. Conocimientos profundos del mercado del software } \\
\text { (SW) bibliotecario y documental } \\
\text { Conocimientos altos sobre las TIC, especialmente en } \\
\text { lo que se refiere a las bibliotecas: } \\
\text { 2.1. Internet y web social } \\
\text { 2.2. Digitalización/OCR } \\
\text { 2.3. Metadatos y datos enlazados } \\
\text { 2.4. Lenguajes de marcado } \\
\text { 2.5. Indexación y bases de datos } \\
\text { 2.6. Diseño web y de interfaces } \\
\text { 2.7. Repositorios } \\
\text { Conocimientos apreciables de SW básico de usuario } \\
\text { 3.1. SW libre } \\
\text { 3.2. Ofimática. } \\
\text { Conocimientos básicos de redes y hardware }\end{array}$ & 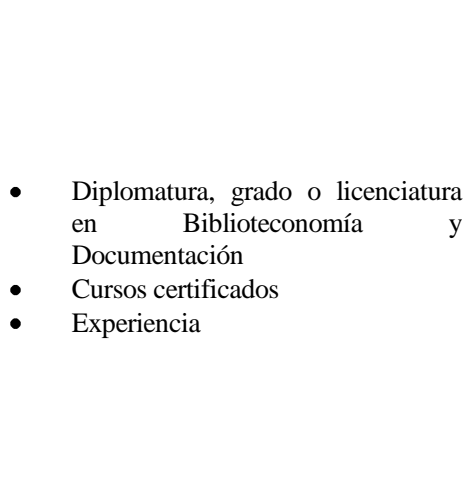 \\
\hline
\end{tabular}


Tabla 4: Competencias bibliotecarias

\begin{tabular}{|c|c|}
\hline Denominación de la competencia & Forma de valoración \\
\hline $\begin{array}{l}\text { 1. Conocimientos bibliotecarios generales } \\
\text { 1.1. Servicios al usuario } \\
\text { 1.2. Proceso técnico } \\
\text { 1.3. Gestión de la colección } \\
\text { 1.4. Gestión documental } \\
\text { 1.5. Recuperación de la información } \\
\text { 1.6. ALFIN/CI2/Digcomp } \\
\text { 1.7. etc. } \\
\text { 2. Conocimiento del mercado editorial } \\
\text { 3. Conocimiento de consorcios } \\
\text { 4. Conocimientos didácticos y de formación } \\
\text { 5. Capacidad de investigación } \\
\text { 6. Conocimientos de propiedad intelectual y derecho } \\
\text { bibliotecario }\end{array}$ & $\begin{array}{l}\text { - Diplomatura, grado o licenciatura } \\
\text { en Biblioteconomía } \\
\text { Documentación } \\
\text { - } \quad \text { Cursos certificados } \\
\text { - } \quad \text { Experiencia } \\
\text { - Para la capacidad de investigación: } \\
\text { doctorado y/o trabajos publicados }\end{array}$ \\
\hline
\end{tabular}

Tabla 5: Cualificación y experiencia profesional

\begin{tabular}{|c|c|c|}
\hline $\begin{array}{l}\text { Clase de } \\
\text { competencia }\end{array}$ & Denominación de la competencia & Forma de valoración \\
\hline $\begin{array}{l}\text { Cualificación } \\
\text { profesional }\end{array}$ & $\begin{array}{l}\text { 1. Pertenencia al cuerpo facultativo de bibliotecas o } \\
\text { similares (ineludible) } \\
\text { 2. Licenciatura (ineludible) } \\
\text { 3. Licenciatura, grado o master en Documentación o } \\
\text { Biblioteconomía (preferible) } \\
\text { 4. Doctorado (preferible) } \\
\text { 5. Conocimientos de idiomas: } \\
\text { 5.1. Inglés (C1 mínimo) } \\
\text { 5.2. Otro(s) idioma comunitario } \\
\text { 5.3. Lengua oficial autonómica }(\mathrm{C} 1)\end{array}$ & $\begin{array}{ll}- & \text { Títulos } \\
\text { - } & \text { Certificados } \\
\text { oficiales } \\
\text { comúnmente } \\
\text { aceptados } \\
\text { (Cambridge, } \\
\text { ACLES, etc.) }\end{array}$ \\
\hline Experiencia & $\begin{array}{l}\text { 1. Experiencia de al menos } 5 \text { años en bibliotecas del } \\
\text { mismo tipo } \\
\text { 2. Experiencia de al menos cinco años en puestos de } \\
\text { gestión }\end{array}$ & $\begin{array}{l}\text { - Certificados de } \\
\text { los servicios de } \\
\text { recursos } \\
\text { humanos }\end{array}$ \\
\hline
\end{tabular}

El problema de las competencias descritas es la valoración de las mismas. Algunas son puramente numéricas, como los años de experiencia, y la mayoría pueden ser valoradas con base al curriculum vitae: certificados, titulaciones, etc. Al respecto, la existencia de escuelas de gobierno permitiría, al menos sobre la teoría, asegurar buena parte de las competencias descritas.

Además, se puede recurrir a consultores externos, expertos en procesos de selección, para valorar el desarrollo de las competencias personales y relacionadas con el liderazgo. Así, sería interesante que el tribunal responsable del proceso contase con ese asesoramiento, sin basarse exclusivamente en el curriculum vitae. Por supuesto, también serían necesarias entrevistas personales con los candidatos preseleccionados. 
Por fin, en lo que se refiere a los conocimientos bibliotecarios, estos, una vez más, pueden ser reconocibles por medio de titulaciones, certificados y experiencia; pero tampoco está de más calibrar su actualización mediante la citada entrevista.

\section{CONCLUSIONES Y PROPUESTAS DE DESARROLLO DE LA INVESTIGACIÓN}

Aunque hasta ahora no se había sugerido la necesidad de crear un perfil de director de biblioteca universitaria en España, las exigencias que plantean las bibliotecas actuales parecen recomendarlo. Recordemos que en países con mayor desarrollo bibliotecario, como EUA, Australia y el Reino Unido, se produjeron en los últimos años un gran número de jubilaciones de directores de bibliotecas, con la consiguiente amplia oferta de puestos, que se encontró con un mercado laboral poco preparado, con escasez de candidatos válidos. Esto llevó a algunas de las universidades más importantes a contratar empresas caza-talentos para buscar la persona adecuada (Lonergan, 2009). En nuestro entorno, estos puestos se suelen nutrir de la oferta interna de cada universidad, pero no siempre manejando criterios estrictamente profesionales. Todo ello abunda en la necesidad señalada.

Por otra parte, para asegurarnos que los candidatos cumplan con el perfil requerido, se deberían articular vías para que estos cuenten con un reconocimiento previo, una certificación u otro método que lo asegure.

El perfil definitivo debería estar avalado por una entidad que, en este caso, tendría que ser REBIUN como organización que aglutina a todas las bibliotecas de este tipo; y con una reconocida y larga trayectoria, además de con experiencia en el desarrollo de normativas y proyectos de apoyo a la gestión.

Como colofón de este trabajo, se considera que se debería modificar el procedimiento de los concursos para este tipo de puestos:

1) La mayoría de los puestos de dirección bibliotecaria se ocupan por el procedimiento de Libre Designación, lo cual habilita a las universidades a autoimponerse unos requisitos mínimos. Este podría proceder de la sectorial CRUE-REBIUN, que mediante un grupo de trabajo podría aprobar un curriculum vitae-tipo y un perfil profesional para estos puestos, al menos orientativo.

2) La selección podría realizarse o al menos supervisarse por entes independientes al organismo contratante: una agencia estatal externa, que podría ser de la propia REBIUN, y que realizase la primera criba sobre los méritos mediante un procedimiento de doble ciego, aun sabiendo que las dimensiones limitadas de la profesión llevarían a una identificación bastante sencilla de los candidatos.

3) Siguiendo las ideas de Blanco Gaztañaga (2010: 202), esta agencia o entidad externa podría proponer una terna o poner las condiciones mínimas para ser elegible. También se podría pensar en la implantación de una habilitación nacional.

4) La creación de Escuelas de Gobierno, y el paso por ellas, certificaría una serie de conocimientos y herramientas básicas a los bibliotecarios que quisieran optar a estos puestos.

5) Por fin, sería interesante repetir este proceso en otros cuerpos y escalas. 


\section{BIBLIOGRAFÍA}

ABELS, E.; JONES, R.; LATHAM, J.; MAGNONI, D. y MARSHALL, J. G. (2003). Competencies for Information Professionals of the 21st Century, Alexandria, VA: Special Library Association (SLA), en https://www.sla.org/about-sla/competencies/

AMERICAN LIBRARY ASSOCIATION (2015) What Library Directors Need to Know, Chicago, IL, ALA, en https://goo.gl/Vt1ce7

ANGLADA, L. (1993). "El futur de les biblioteques i el bibliotecari del futur”, en Ítem, 13: 13-24, en http://www.raco.cat/index.php/Item/article/view/22428/22262

ARABELLA ADVISORS. (2015). Cultivating Global Library Leadership: A Review of Leadership Training Programs for Librarians Worldwide. San Francisco, CA: Arabella, en http://tascha.uw.edu/publications/cultivating-global-library-leadership

BLANCO, C. (2010). "La figura del directivo público profesional: reclutamiento y estatuto", en DA. Revista Documentación Administrativa, 286-287: 179-205, en https://goo.gl/HRW0nm

CANALES, J. M. (2012). "Algunas consideraciones sobre los nuevos retos de la modernización del control de las administraciones públicas contemporáneas”, en Auditoría Pública, 58: 19-29.

- (2015). Estudios sobre la democracia, el gobierno y la administración pública contemporánea. Madrid: Universitas.

CARPENTER, M. T. H. (2012). "Cheerleader, Opportunity Seeker, and Master Strategist: ARL Directors as Entrepreneurial Leaders", en College \& Research Libraries,73 (1): 11-32, en http://dx.doi.org/10.5860/crl-192

CHOW, A. S. y RICH, M. (2013). "The ideal qualities and tasks of library leaders: Perspectives of academic, public, school, and special library administrators", en Library Leadership and Management, 27: 1-2, en https://goo.gl/nz4aLp

CORRALES, R. (2011). "Nueva gerencia en la carrera pública: Meritocracia vs. Padrinocracia, en Justicia y derecho, 4 (6) en https://goo.gl/Rc96kr

CUADRADO, D. (2009). "Progresiva desprofesionalización de la Administración Pública", en Actualidad administrativa, 11: 2 .

DELONG, K. (2009). "The Engagement of New Library Professionals in Leadership", en The Journal of Academic Librarianship, 35 (5): 445-456, en http://dx.doi.org/10.1016/j. acalib.2009.06.006

ECIA (European Council of Information Associations) (2004). Euroreferencial en Información y Documentación: Volumen 1. Competencias y aptitudes de los profesionales europeos de información y documentación. Madrid: SEDIC, en http://www.certidoc.net/es1/euref1-espanol.pdf

FESABID: Federación Española de Sociedades de Archivística, Biblioteconomía, Documentación y Museística (2007). Propuesta de Fesabid sobre política de bibliotecas y de archivos a los partidos políticos para su incorporación en los programas electorales y de gobierno, 15 de diciembre. Madrid: FESABID, en https://goo.gl/HP8Ye4

FGSR: Fundación Germán Sánchez Ruipérez (2015). "Está disponible el informe de la Fundación Gates sobre la formación para líderes en bibliotecas", en Lectura Lab. [blog], 2 de marzo, en https://goo.gl/w1J7OY

GALLO-LEÓN, J. P., ANGLADA, L., QUÍLEZ, P., NOVOA, C., GÓMEZ, L., ELIZARI, J. F.,... DOCAMPO, J. (2014). Prospectiva 2020: Las diez áreas que más van a cambiar en nuestras bibliotecas en los próximos años, Madrid, Consejo de Cooperación Bibliotecaria, Ministerio de Cultura, en http://hdl.handle.net/10421/7460

GARCÍA, J. y SUTHERLAND, S. (2001). Directores de biblioteca pública en la arena política. Barcelona: Fundación Bertelsmann, en https://goo.g1/7p8gNa

GOLEMAN, D.; BOYATZIS, R. y MCKEE, A. (2002). Primal Leadership. Boston, MA: Harvard Business School Press.

GRIMES, M. F. y GRIMES, P. W. (2008). "The Academic Librarian Labor Market and the Role of the Master of Library Science Degree: 1975 through 2005" en The Journal of Academic Librarianship, 34 (4): 332-339, en http://dx.doi.org/10.1016/j.acalib.2008.05.023

HERNON, P. (2011). "Becoming a university library director", en Library \& Information Science Research, 33 (4): 276-283, en http://dx.doi.org/10.1016/j.lisr.2011.04.003 
HERNON, P. y SCHWARTZ, C. (2008). "Reflections on a New Ph.D. Program: Thoughts of Interest to the Profession", en The Journal of Academic Librarianship, 34 (1):1-2, en http://dx.doi.org/10.1016/j.acalib.2007.11.004

- (2011). "The preparation of leaders in library and information science", en Library \& Information Science Research, 33 (4): 276-283, en http://dx.doi.org/10.1016/j.lisr.2011. 07.002

IRURZUN, F. (2010). "Ética y responsabilidad en la Administración Pública", en DA. Revista Documentación Administrativa, 286-287: 79-111, en https://goo.gl/QWV1m1

JOAO, M.; EXTREMEÑO, A. I. y FIRMINO, A. (2012). Una nueva biblioteca para una nueva universidad. Gijón: Trea.

JORDAN, M. W. (2012). “Developing leadership competencies in librarians”, en IFLA Journal, 38 (1): pp. 37-46, en http://dx.doi.org/10.1177/0340035211435074

KREITZ, P. A. (2009). "Leadership and Emotional Intelligence: A Study of University Library Directors and Their Senior Management Teams", en College \& Research Libraries, 70 (6): 531-554, en http://dx.doi.org/10.5860/crl.70.6.531

LONERGAN, D. (2009). "Have ability, won't travel", en Community \& Junior College Libraries, 15 (4): 217-222, en http://dx.doi.org/10.1080/02763910903239997

LONGO, F. (2010). "Hacia una nueva meritocracia pública", en Factor Humà [Tomado de un artículo aparecido en La Vanguardia, Domingo, 12 de diciembre de 2010], en https://goo.gl/FaScTY

MALONEY, K.; ANTELMAN, K.; ARLITSCH, K. y BUTLER, J. (2010). "Future Leaders' Views on Organizational Culture", en College \& Research Libraries, 71 (4): 322-347, en http://dx.doi.org/10.5860/crl-47

MARTÍNEZ, A. M. (2013). "La necesaria innovación en la administración pública, Abril Abadín... [et al.]" [Recensión], en RIPS: Revista de Investigaciones Políticas y Sociológicas, 12 (3): 165-172, en http://www.usc.es/revistas/index.php/rips/article/view/1636

MOREIRO, J. A. (1999). "Ajuste de los licenciados en documentación a la oferta de trabajo. Observaciones hechas en la universidad Carlos III de Madrid", en Revista General de Información y Documentación, 9 (2): 13-28.

NEWCOMBE, P. A. y DONOVAN, J. M. (2013). "Becoming director: an internal candidate's view", en Library Management, 34 (3): 188-199, en http://dx.doi.org/10.1108/01435121311310888

OCDE (2008). The State of the Public Service. Paris: OECD Publishing.

SÁNCHEZ, J. A. (2007). "El Servicio Público Canadiense bajo los mandatos de Brian Mulroney y Jean Chrétien", en D. Montero; y R. Rodríguez (Comps.) Políticas públicas, relaciones bilaterales $e$ Identidad en Canadá. Una nueva connotación en el inicio del siglo XXI. La Habana, Cuba: Félix Varela, pp. 7-28.

TAYLOR, V. y RENZULLI, G. (1997). "Mastering middle management", en Information Outlook, 1 (2): 18, en http://search.proquest.com/docview/57404801?accountid=17225

TEJADA, C. M. y MARTÍNEZ, B. (Coords.) (2013). Perfiles profesionales del Sistema Bibliotecario Español: fichas de caracterización. Consejo de Cooperación Bibliotecaria. Grupo de Trabajo de Perfiles Profesionales (2013). Madrid: Ministerio de Educación, Cultura y Deporte, en https://goo.gl/UYJz6k

WEINGAND, D. (1999). "Gestión de la biblioteca pública actual: dirección y liderazgo", en Boletín de la Asociación Andaluza de Bibliotecarios, 14 (56): 59-77, en http://eprints.rclis.org/5989/

WHITE, H. S. (1997). "Should leaders want to be managers and give up all that freedom?", en Library Journal, 122 (15):48, en http://search.proquest.com/docview/57430237?accountid

$=17225$

YOUNGER, P. (2008). “CPD for library leadership: Some practical suggestions”, en Impact: Journal of the Career Development Group, 11 (3): 46-49, en http://search.proquest.com/ docview/57688695?accountid=17225

\section{ANEXO. Ofertas de empleo consultadas}

-Alamo Colleges. Director of Library Services https://goo.gl/i8EpWC

-Binghamton University https://goo.gl/53rM8j 
-California State University http://csucareers.calstate.edu/Detail.aspx?pid=48212

-Corte Internacional de Justicia: https://goo.gl/pS3zSW

-Drew University https://goo.gl/p3hrb7

-European University Institute https://goo.gl/qHBuhr

-Fairfield University. Dean of Libraries \& University Librarian https://goo.gl/RXr7CA

-Florida Atlantic University (FAU) https://goo.gl/hfLOLA

-Georgia State University https://goo.gl/P6iSlj

-Kansas Wesleyan http://www.kwu.edu/job-description-director-of-library-services

-Louisvelle University Libraries https://goo.gl/1NzjH2 (no es oferta, sólo descripción del trabajo)

-San Jose State University. Dean, University Library https://goo.gl/PPJpXu

-Spalding University http://joblist.ala.org/modules/jobseeker/controller.cfm

-Texas A\&M University-Central Texas https://goo.gl/ixKs7V

-Texas Woman's University. Dean, Libraries https://goo.gl/ZuyjHA

-The Bishop's School. Library Director https://goo.gl/mljLa6

-University of Massachusetts Amherst. Dean of Libraries https://goo.gl/X4BYJH

-University of Virginia. Dean of Libraries https://goo.gl/Nu7ph8

-Vanderbilt University https://goo.gl/QehR9O

-Washington State University Tri-Cities https://www.wsujobs.com/postings/18695

-Weill Medical College of Cornell University https://goo.gl/kVh14O

\section{Breve currículo:}

\section{José Pablo Gallo León}

Doctor por la Universidad de Murcia en Comunicación y Documentación; licenciado en Geografía e Historia (Historia del Arte) y Magíster en Biblioteconomía por la Universidad Complutense de Madrid; y Especialista universitario en Dirección Pública por la Universidad de Alicante. Ha sido director de la Biblioteca Regional de Murcia y de la Biblioteca de la Universidad Miguel Hernández. Actualmente trabaja en la Universidad de Alicante como bibliotecario y da clases como profesor asociado en la Universidad de Murcia, Departamento de Información y Documentación. Su campo de investigación se orienta hacia la gestión bibliotecaria: servicios e infraestructuras; y prospectiva sobre bibliotecas. Ha participado y coordinado grupos de trabajo de REBIUN y Cooperación Bibliotecaria, siendo el más reciente el Grupo estratégico para el estudio de prospectiva sobre la biblioteca del CCB. 